\title{
Hadron multiplicity induced by top quark decays at the LHC
}

\section{Roman Ryutin*}

IHEP

E-mail: Roman.Rioutinedcern.ch

The average charged hadron multiplicities induced by top quark decays are calculated in pQCD at LHC energies. Different modes of top production are considered. Proposed measurements can be used as an additional precise test of pQCD independent on a fragmentation model.

The XXth International Workshop High Energy Physics and Quantum Field Theory September 24 - October 1, 2011

Sochi, Russia

${ }^{*}$ Speaker. 


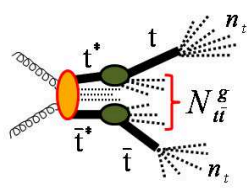

a)

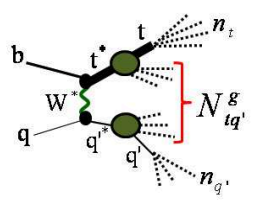

c)

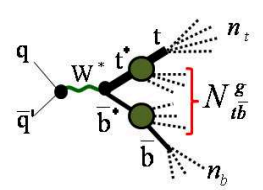

b)

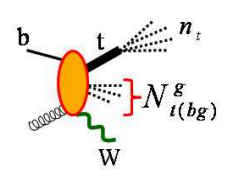

d)

Figure 1: Parton level processes of top production at LHC considered in this paper: a) dominant $t \bar{t}$ production in the gluon-gluon fusion; b) s-channel single top production; c) t-channel single top production; d) tW production.

\section{Introduction}

Top physics is one of the most popular topics now, since LHC can be used as a top-factory. The unique characteristics of the top quark like large mass, short life-time, almost exclusive decay to $\mathrm{b}$ and $\mathrm{W}$, allow us to to use top events for direct verification of $\mathrm{pQCD}$. There are many tests of QCD based on measurements of jets, high transverse momentum particles and event shapes. But, usually in the final step we need some fragmentation model to simulate parton to hadrons transition. It was shown in previous works [1]-[4], that measurements of average charged multiplicity in a jet (especially jet produced in heavy quark decay) can serve as a precise test of pQCD independent on a fragmentation model. Authors considered $e^{+} e^{-}$annihilation with heavy quark production. There are some useful properties of top production in the process $e^{+} e^{-} \rightarrow t \bar{t}$. First of all, we can assume independent fragmentation of final on-shell top quarks. This is analogous to $W^{+} W^{-}$ production, which was confirmed experimentally [5],[6]. In this case $t \bar{t}$ system is a color singlet. And heaviness simplify much theoretical calculations. Using numbers of primary multiplicies, phenomenological gluon multiplicity fixed by low energy data and pQCD calculations, it is possible to estimate average charged multiplicity in top events.

\section{Model}

The model used in this section is analogous to the one presented in [1]. Here we consider processes of the type $p+p \rightarrow t \bar{t}+X$ or $p+p \rightarrow t+X$ (see Fig. 1). The basic formula for inclusive production of the system $M$ in the collinear approximation looks as follows

$$
\frac{d \sigma_{p p \rightarrow M X}(s)}{d x_{1} d x_{2} d \Phi_{M}}=\sum_{i, j} f_{i}\left(x_{1}\right) f_{j}\left(x_{2}\right) \frac{d \hat{\sigma}_{i j \rightarrow M}}{d \Phi_{M}}\left(x_{1} x_{2} s ;\left\{\Phi_{M}\right\}\right),
$$

where $f_{i}(x)$ is the probability to find parton $i$ (quark, anti-quark or gluon) with the longitudinal momentum $x \sqrt{s} / 2$ in a proton. Renormalization and factorization scales are hidden in $f$ and $\hat{\sigma}$. Here $\mathrm{M}$ is the system of different final states like $t \bar{t}, t \bar{b}, t q, t W$, which corresponds to different 
mechanisms of the inclusive top production at the LHC. $X$ includes beam remnants $\left(X_{\text {beam }}\right)$ and also the secondary radiation induced by color interactions inside $\mathrm{M}\left(X_{M}\right)$ plus possible interaction between $\mathrm{M}$ and beam remnants $\left(X_{M \text {-beam }}\right)$, if $\mathrm{M}$ is not a color singlet. Variables $x_{1,2}$ are fixed in every separate event and can be calculated experimentally. Usually the sum in (2.1) can be approximated by a single factorized term which includes parton distributions multiplied by the amplitude squared of parton-parton cross-sections: $g+g \rightarrow t \bar{t}+X_{M}$ (dominates at the LHC), $q+\bar{q}^{\prime} \rightarrow W^{*} \rightarrow t \bar{b}+X_{M}, b+q \rightarrow t q^{\prime}+X_{M}, b+g \rightarrow t W+X_{M}$, where $q, q^{\prime}$ denotes corresponding light quarks. For our purposes it is enough to consider initial parton collisions instead of pp process, since we have to calculate only the charged hadron multiplicity of the $M$ plus $X_{M}$ in a separate event. Complications concerning $X_{\text {beam }}$ and $X_{M \text {-beam }}$ are discussed below. $X_{M}$ comes from virtual gluon radiation.

For the average multiplicity of hadrons in $M+X_{M}$ we have:

$$
\begin{aligned}
& N_{M+X_{M}}^{h}\left(Q^{2}\right)=n_{M}+N_{M}^{g} \equiv n_{M}+\int \frac{d^{4} k}{(2 \pi)^{4}} \Pi\left(Q^{2}, k^{2}, k q_{1}, k q_{2}\right) \frac{n_{g}\left(k^{2}\right)}{k^{2}} \\
& \Pi\left(Q^{2}, k^{2}, k q_{1}, k q_{2}\right)=\left(-g^{\mu v}\right) \delta_{a b}\left[\prod_{i=1}^{N_{\text {in..gl. }}} \tilde{d}^{\rho_{i} \sigma_{i}}\left(q_{i}, n\right) \delta_{a_{i} b_{i}}\right] \times \Pi_{\left\{\rho_{i} \sigma_{i}\right\} ; \mu v}^{\left\{a_{i} b_{i}\right\} ; a b}\left(q_{1}, q_{2}, k\right), \\
& k^{\mu} \Pi_{\mu v}^{a b}\left(q_{1}, q_{2}, k\right)=0(\text { see [9]) } \\
& d_{a b}^{\mu v}(k) \equiv i \tilde{d}_{a b}^{\mu v}(k) /\left(k^{2}+i 0\right), \\
& \tilde{d}^{\rho_{i} \sigma_{i}}\left(q_{i}, n\right)=\sum_{\lambda=1,2} \varepsilon_{(i) ; \lambda}^{\rho_{i}} \varepsilon_{(i) ; \lambda}^{* ; \sigma_{i}}=-g^{\rho_{i} \sigma_{i}}+\frac{q_{i}^{\rho_{i}} n^{\sigma_{i}}+n^{\rho_{i}} q_{i}^{\sigma_{i}}}{q n}-\frac{n^{2}}{q n^{2}} q_{i}^{\rho_{i}} q_{i}^{\sigma_{i}}
\end{aligned}
$$

Here $\prod_{\left\{\rho_{i} \sigma_{i}\right\} ; \mu v}^{\left\{a_{i} b_{i}\right\} ; a b}\left(q_{1}, q_{2}, k\right)$ can be calculated in the first order in the strong coupling constant as the amplitude squared of the corresponding process with $X_{M}=g$ normalized to the total rate of the process without $X_{M}, N_{\text {in.gl. }}=0,1,2$ is the number of initial gluons, $q_{1,2}$ are momenta of initial partons, $\varepsilon_{(i) ; \lambda}^{\rho} q_{i ; \rho}=0, q_{i}^{2}=0, n$ is an appropriate four-vector in the corresponding process. $(a, b)$ and $\left(a^{\prime}, b^{\prime}\right)$ denote color indices, and $Q=\sqrt{\left(q_{1}+q_{2}\right)^{2}}$ is the energy of colliding partons. Concrete form of the function $\Pi$ for different processes can be found in [10].

The term $n_{M}$ in Eq. (2.2) is the multiplicity from the fragmentation of leading particles in the final state. For example, in the process $g+g \rightarrow t \bar{t}+X_{M}$ (see Fig. 1a) $n_{M}=n_{t \bar{t}}=2 n_{t}$, where $n_{t}$ was calculated in [2]. In other processes $n_{M}$ is appropriate combination of multiplicities which are taken from the analysis of data and pQCD calculations:

$$
\begin{aligned}
& n_{t}^{H} \equiv n_{t}(t \rightarrow \text { hadrons })=41.03 \pm 0.54, n_{t}^{L} \equiv n_{t}\left(t \rightarrow l \bar{v}_{l}+\text { hadrons }\right)=21.9 \pm 0.53[2] \\
& n_{W}(W \rightarrow \text { hadrons })=19.34 \pm 0.10[2], n_{c}=2.6, n_{b}=5.5[7], n_{q}=1.2[8]
\end{aligned}
$$

The dimensionless quantity $n_{g}\left(k^{2}\right)$ describes the average multiplicity of hadrons in the gluon jet with the virtuality $k^{2}$. It is gauge invariant and depends only on the virtuality $k^{2}$. This quantity cannot be calculated perturbatively. It is usually assumed that the average hadron multiplicity is proportional to $n_{g}\left(k^{2}, Q_{0}^{2}\right)$, i.e. the average multiplicity of (off-shell) partons with the "mass" $Q_{0}$ (the so-called local parton-hadron duality):

$$
n_{g}\left(k^{2}\right)=n_{g}\left(k^{2}, Q_{0}^{2}\right) K\left(Q_{0}^{2}\right)
$$


where $K\left(Q_{0}^{2}\right)$ is a phenomenological energy-independent factor. The QCD evolution equation for $n_{g}\left(k^{2}, Q_{0}^{2}\right)$ is derived in [1]. We use the "conventional standard" value $Q_{0}=1 \mathrm{GeV}$ for further numerical calculations.

In this paper we will use two phenomenological expressions (to estimate theoretical uncertainties) for $n_{g}$ which can be found in [1]:

$$
\begin{aligned}
& n_{g}^{i}\left(k^{2}\right)=k^{2} \frac{d}{d k^{2}} N_{g}^{i}\left(k^{2}, Q_{0}^{2}\right), \\
& N_{g}^{1}\left(k^{2}\right)=3.89+0.01 \mathrm{e}^{1.63 \sqrt{\ln \left(\frac{k^{2}}{(0.87 \mathrm{GeV})^{2}}\right)}, N_{g}^{2}\left(k^{2}\right)=4.21+0.012 \ln ^{2} \frac{k^{2}}{(0.93 \mathrm{GeV})^{2}},}
\end{aligned}
$$

where $N_{g}^{i}\left(k^{2}, Q_{0}^{2}\right)$ is the average multiplicity from the gluon jet whose virtuality $p^{2}$ varies up to $k^{2}$. Very often $N_{g}\left(k^{2}\right)$ is erroneously called the average multiplicity of the gluon jet with fixed virtuality $k^{2}$. This meaning should be addressed to $n_{g}\left(k^{2}\right)$ only.

\section{Numerical results}

In this section we consider numerical results for average charged multiplicities in different processes of top production at the LHC. Below we consider the phase space when final jets have low transverse momentum cuts $P_{t}$, and the final gluon jet can not be experimentally separated from one of final quark jets (i.e. gluon jet lies within the cone $\cos \theta_{g q}>R=0.9$, where $t$ thet $a_{g q}<0.45$ is the angle between the gluon and quark jets).

Let us begin with the inclusive $t \bar{t}$ production (Fig. 1a). The total cross-section of the inclusive process $p p \rightarrow t \bar{t}+X$ is about $833 \mathrm{pb}$ at $14 \mathrm{TeV}$. In this article we consider only the gluon-gluon fusion mechanism of this process since at LHC it is dominant. Numerical values for $N_{t \bar{t}}^{g}$ are shown on the Fig. 2. Here and below theoretical errors are estimated by the use of two different parametrizations (2.11) for the hadronic multiplicity in a gluon jet. The average charged multiplicity in different decay modes (hadronic, semileptonic and leptonic) can be calculated as follows

$$
\begin{aligned}
& N_{t \bar{t} \rightarrow \text { hadrons }}^{h}(Q)=2 n_{t}^{H}+N_{t \bar{t}}^{g}(Q), N_{t \bar{t} \rightarrow l \bar{l}_{l}+\text { hadrons }}^{h}(Q)=n_{t}^{H}+n_{t}^{L}+N_{t \bar{t}}^{g}(Q), \\
& N_{t \bar{t} \rightarrow l^{+} l^{-} v_{l} \bar{v}_{l}+\text { hadrons }}^{h}(Q)=2 n_{t}^{L}+N_{t \bar{t}}^{g}(Q) .
\end{aligned}
$$

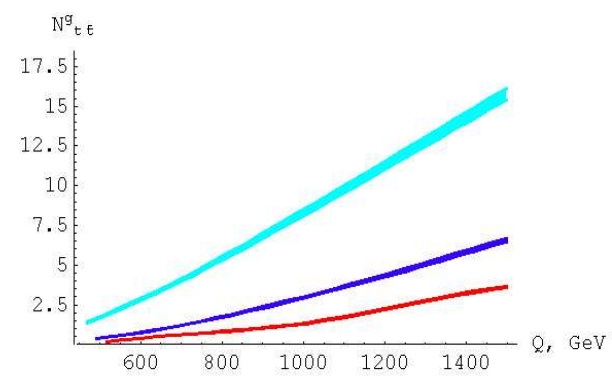

Figure 2: $t \bar{t}$ production. Multiplicity $N_{t \bar{t}}^{g}$ versus $Q$ for different cuts of jet transverse momenta. Top-down: $P_{t}^{j e t}>10 \mathrm{GeV} \rightarrow P_{t}^{j e t}>30 \mathrm{GeV} \rightarrow P_{t}^{j e t}>50 \mathrm{GeV}$. 
As you see on the Fig. 2, the energy dependence of $N_{t \bar{t}}^{g}$ is visible. In this work we assume that color reconnection of $t \bar{t}$ and beam remnants is small due to the strong suppression of this processes with high transverse momentum transfer (typical jet transverse momentum cut at the LHC is about $20-40 \mathrm{GeV}$ ). Also $t$ and $\bar{t}$ fragment independently after the interaction inside the $t \bar{t}$ system. It looks similar to the process of $W^{+} W^{-}$fragmentation in $e^{+} e^{-}$annihilation. The effect of possible color reconnection was investigated by comparing hadronic multiplicities in $e^{+} e^{-} \rightarrow W^{+} W^{-} \rightarrow q \bar{q}^{\prime} q \bar{q}^{\prime}$ and $e^{+} e^{-} \rightarrow W^{+} W^{-} \rightarrow q \bar{q}^{\prime} l \bar{v}_{l}$. No evidence for final state interactions was found by measuring the difference $<n_{4 q}^{h}>-2<n_{2 q l \bar{v}}^{h}>$ [5],[6]. The values for average charged multiplicities can be compared with the present LHC data on the inclusive $t \bar{t}$ production.

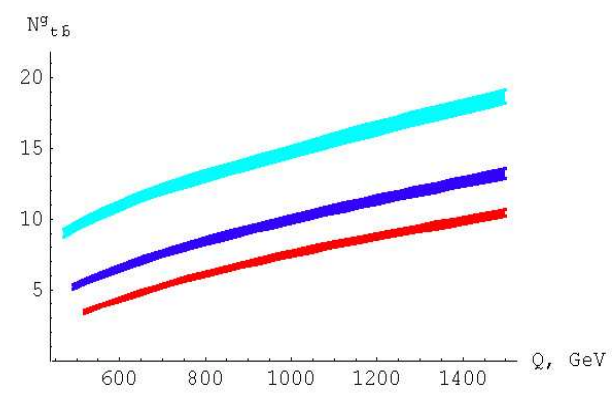

Figure 3: S-channel single top production. Multiplicity $N_{t \bar{b}}^{g}(Q)$ versus $Q$ for different cuts of jet transverse momenta. Top-down: $P_{t}^{\text {jet }}>10 \mathrm{GeV} \rightarrow P_{t}^{\text {jet }}>30 \mathrm{GeV} \rightarrow P_{t}^{\text {jet }}>50 \mathrm{GeV}$.

The case of s-channel single top production (Fig. 1b) is close to the $e^{+} e^{-}$one, since the final state is a result of $W$ decay, i.e. color singlet. That is why we have no color reconnection with beam remnants. However, the cross-section of this process is rather small (about $11 \mathrm{pb}$ at $14 \mathrm{TeV}$ ), and the experimental task on the extraction of the multiplicity looks more difficult than, for example, in t-channel single top or $t \bar{t}$ production. Numerical values for $N_{t \bar{b}}^{g}$ are shown on the Fig. 3 . The average charged multiplicity in different decay modes can be calculated as follows

$$
N_{t \bar{b} \rightarrow \text { hadrons }}^{h}(Q)=n_{t}^{H}+n_{b}+N_{t \bar{b}}^{g}(Q), N_{t \bar{b} \rightarrow l \bar{l}_{l}+\text { hadrons }}^{h}(Q)=n_{t}^{L}+n_{b}+N_{t \bar{b}}^{g}(Q) .
$$

The energy dependence is not so strong as in the previous case (see Fig. 3).

The process of $\mathrm{t}$-channel single top production $p p \rightarrow t+X$ has higher rate (about $245 \mathrm{pb}$ at $14 \mathrm{TeV}$ ) than the previous one, but we have to make the same assumptions concerning fragmentation and color reconnection processes as in $t \bar{t}$ production. Here results for the parton level process (Fig. 1c) are presented. Numerical values for $N_{t q^{\prime}}^{g}$ are shown on the Fig. 4. The average charged multiplicity in different decay modes looks as follows

$$
N_{t q^{\prime} \rightarrow \text { hadrons }}^{h}(Q)=n_{t}^{H}+n_{q}+N_{t q^{\prime}}^{g}(Q), N_{t q^{\prime} \rightarrow l \bar{v}_{l}+\text { hadrons }}^{h}(Q)=n_{t}^{L}+n_{q}+N_{t q^{\prime}}^{g}(Q) .
$$

As you can see on the Fig. 4, the value of $N_{t q^{\prime}}^{g}$ is rather small in the wide kinematical region, and energy dependence is not strong. It is important for the estimation of the multiplicity from beam remnants plus color reconnection effects, since values $n_{t}^{H}, n_{t}^{L}, n_{q}$ are fixed by previous measurements and $N_{t q^{\prime}}^{g}(Q) \ll n_{t}$. From this point of view the t-channel single top production looks the most interesting process for the multiplicity measurements. 


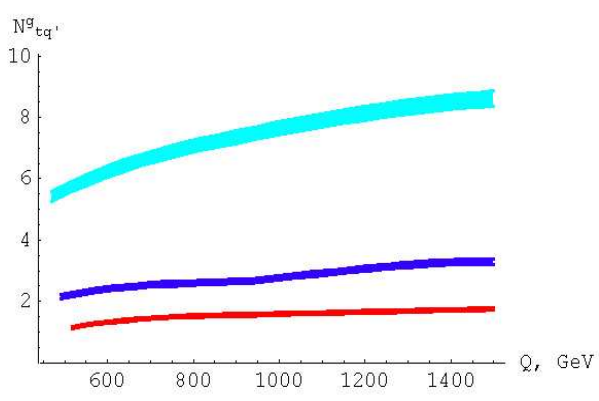

Figure 4: T-channel single top production. Multiplicity $N_{t q^{\prime}}^{g}(Q)$ versus $Q$ for different cuts of jet transverse momenta. Top-down: $P_{t}^{\text {jet }}>10 \mathrm{GeV} \rightarrow P_{t}^{j e t}>30 \mathrm{GeV} \rightarrow P_{t}^{j e t}>50 \mathrm{GeV}$.

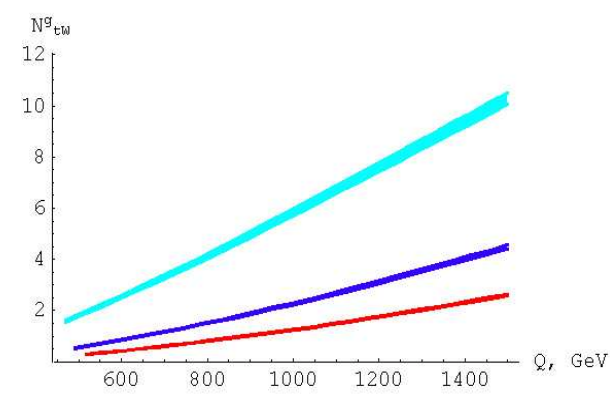

Figure 5: $t W$ production. Multiplicity $N_{t W}^{g}(Q)$ versus $Q$ for different cuts of jet transverse momenta. Top-down: $P_{t}^{j e t}>10 \mathrm{GeV} \rightarrow P_{t}^{j e t}>30 \mathrm{GeV} \rightarrow P_{t}^{j e t}>50 \mathrm{GeV}$.

$t W$ production has intermediate cross-section of the order $62 \mathrm{pb}$ at $14 \mathrm{TeV}$ which lies between s- and t-channel single top production rates. Probably, specific signature of this process would help in the measurements proposed in this work. Numerical values for $N_{t W}^{g}$ are shown on the Fig. 5. The process of $t W$ production (Fig. 1d) has 3 decay modes. The corresponding average charged multiplicities are

$$
\begin{aligned}
& N_{t W \rightarrow \text { hadrons }}^{h}(Q)=n_{t}^{H}+n_{W}+N_{t W}^{g}(Q), N_{t W \rightarrow(W) l \bar{v}_{l}+\text { hadrons }}^{h}(Q)=n_{t}^{H}+N_{t W}^{g}(Q), \\
& N_{t W \rightarrow l^{+} l^{-} v_{l} \bar{v}_{l}+\text { hadrons }}^{h}(Q)=n_{t}^{L}+N_{t W}^{g}(Q)
\end{aligned}
$$

The energy dependence is also visible and can be used to test QCD calculations.

\section{Discussions and conclusions}

It is proposed to extract average charged multiplicity of hadrons in "t-induced-jets" in singletop and top anti-top events. At LHC the experimental situation is more difficult than in $e^{+} e^{-}$ annihilation, since jet partons can interact with beam remnants, but: a) in s-channel single top the situation is similar to $e^{+} e^{-}$( $W^{*}$ is a color singlet); b) in other processes we can take jets with high transverse momenta, and such interaction will be suppressed; c) multiplicities $n_{b}, n_{W}, n_{t}, n_{q}$ are fixed from previous calculations and data fitting at low energies; d) $N_{M}^{g}$ are calculated in QCD, 
where $N_{g}$ are independent on a fragmentation model and fixed by data fitting at low energies (Local Parton-Hadron Duality).

There are important tasks that could be solved by multiplicity measurements: to test QCD calculations independently on fragmentation models, to check independent fragmentation of heavy quarks, to check parton-parton C.M. energy dependence of hadron multiplicities, to estimate multiplicity from beam remnants plus from color reconnection effects in t-channel single top for further use in other processes. We can calculate also the difference $\Delta N_{Q q} \equiv N_{Q}-N_{q}$ to cancel effects of color reconnection and beam remnants.

The final experimental task is to extract number of tracks in jets which are produced in top quark decays. To estimate experimental efficiencies and dependence on a fragmentation model we can use any MC generator for top production. At the same time with the top-mass reconstruction procedure (in hadronic mode) we could extract number of tracks which are included into hadronic cluster from single top or top anti-top decays. At the moment we have a good chance to make the new independent test of QCD by the use of recent LHC data at $7 \mathrm{TeV}$. Other experimental aspects of such measurements will be discussed in futher works.

\section{Acknowledgments}

Author thanks V.A. Petrov, A.V. Kisselev, R. Chierici, J. Andrea and S. Wimpenny for fruitful discussions and useful comments.

\section{References}

[1] A.V. Kisselev and V.A. Petrov, Multiple hadron production in $e^{+} e^{-}$annihilation induced by heavy primary quarks: New analysis, Phys. Part. Nucl. 39 (2008) 798.

[2] A.V. Kisselev and V.A. Petrov, Hadron multiplicity in e+e- events induced by top quark pair at the ILC energy, PMC Phys. A 2 (2008) 3.

[3] A.V. Kisselev and V.A. Petrov, Hadron multiplicities in $e^{+} e^{-}$annihilation with heavy primary quarks, Eur. Phys. J. C 50 (2007) 21.

[4] A.V. Kisselev and V.A. Petrov, On hadron multiplicities in $e^{+} e^{-}$events induced by massive quarks, Z. Phys. C 66 (1995) 453.

[5] OPAL Collab., G. Abbiendi et al., Color reconnection studies in $e^{+} e^{-} \rightarrow W^{+} W^{-}$at $\sqrt{s}=183 \mathrm{GeV}$, Phys. Lett. B 453 (1999) 153.

[6] DELPHI Collab., P. Abreu et al., Charged and identified particles in the hadronic decay of $W$ bosons and in $e^{+} e^{-} \rightarrow q \bar{q}$ from $130 \mathrm{GeV}$ to $200 \mathrm{GeV}$., Eur. Phys. J. C 18 (2000) 203.

[7] B.A. Schumm, Yu.L. Dokshitzer, V.A. Khoze, and D.S. Koetke, MLLA and the average charged multiplicity of events containing heavy quarks in $e^{+} e^{-}$annihilation., Phys. Rev. Lett. 69 (1992) 3025.

[8] DELPHI Collab., J. Chrin et al., A study of the difference in charged multiplicity between bottom and light quark initiated events, in proceedings of the 27-th International Conference on High Energy Physics, Glasgow, UK, 20-27 July 1994,p. 893.

[9] E. Leader, E.Predazzi, A note on the implications of gauge invariance in $Q C D$, [arXiv:1101.3425].

[10] R. Ryutin, Hadron multiplicity induced by top quark decays at the LHC, [arXiv: 1105.4776] 